\title{
Investigation on the Dispersion Characteristics in Optical Fiber Telecommunication
}

\author{
Ni Yan Rong and Min Ru \\ Henan Mechanical and Electrical Engineering College, Henan, China, 453000 \\ ABCNIYANRONG@163.COM
}

\begin{abstract}
Dispersion is widely existed in the optical fiber. The reason is various, such as material dispersion, waveguide dispersion, mode dispersion, and so on. It will lead to the signal waveform distortion and pulse broadening and then limit the transmission capacity and bandwidth in the optical fiber. With the rapid development, transmission rate can reach even hundreds of $\mathrm{Gb} / \mathrm{s}$, and it may lead to many problems. Correspondingly, compensation methods have been paid much attention, and there are also many methods proposed for the compensation. However, we should know the characteristics of the dispersion in order to get compensation algorithms with much higher efficiency. Optical fiber systems with better performance will be designed and manufactured. In this paper, appropriate methods are adopted to analyze the photonic crystal fiber. With the adjusting of the structure parameters, we can design a highly nonlinear photonic crystal fiber with different dispersion characteristics. We study the characteristics of the dispersion of optical fiber communication. According to the analysis to obtain the dispersion characteristics of optical fiber under different parameters.
\end{abstract}

Keywords: simulation, optical fiber, dispersion characteristics, telecommunication

\section{Introduction}

With different frequencies of the signal transmitted in optical fiber and various model components of the signal energy, signal waveform distortion and pulse broadening are called dispersion due to the different group velocities in the transmission process. Fiber dispersion limits the transmission capacity and transmission bandwidth of optical fiber [13]. From the mechanism, fiber dispersion is divided into the material dispersion [4-6], waveguide dispersion [7-9], and mode dispersion [10-13]. The first two kinds of dispersion are due to the different frequencies, and the latter dispersion is due to the different modes.

In order to solve the dispersion on the distance and the rate of communication constraints, it must know the characteristics of the dispersion compensation of optical fiber [14-18]. In addition, with the rapid development of optical fiber telecommunication and dispersion compensation scheme, the transmission rate of some high-speed system has reached dozens or even hundreds of $\mathrm{Gb} / \mathrm{s}$. Then, the influence of polarization mode dispersion cannot be ignored [19].

At present, there are many groups' velocity dispersion compensation schemes have been proposed, such as the post dispersion compensation technology, pre dispersion compensation technology, dispersion compensation filter, high dispersion compensation fiber (DCF) technology and optical solution communication technology. The post dispersion compensation technique [20-22] is compensating pulse broadening induced by fiber dispersion in optical signal receiving end through electronic technology. This method is often used for coherent optical fiber communication system, and suitable for low code rate communication system. Its transmission distance is just a few of the 
dispersion length. The pre dispersion compensation technology mainly includes pre chirp technology, complete frequency modulation technology, double binary coding technology, amplifier induced chirp and fiber induced chirp technology. Any kind of pre dispersion compensation technology will produce positive end chirp before get into the optical fiber in order to generate a positive chirp. Filter dispersion compensation [23-25] is based on interference. However, relatively high loss and narrow bandwidth is the limit for its application, and it is quite sensitive and relatively narrow bandwidth of the input light.

In optical fiber transmission, signal transmission rates of different frequencies are different, and there will be different time delay $\tau$ after the same transmission distance, which will lead to different $\Delta \tau$. The bigger time delay difference, the more seriously of the dispersion. The specific performance is that the optical pulse has been broadening in the transmission process in the optical fiber. So the dispersion is always measured with the group delay per unit length.

The basic principle of dispersion compensation for the optical fiber transmission is offset the dispersion by one or more large negative dispersion to compensate the accumulated dispersion, and. /then the total amount of dispersion of the system will be decreased. The most conventional methods of dispersion compensation are: dispersion compensation fiber (DCF), chirped fiber grating, electronic dispersion compensation technology, and so on.

As we know, compensation method has been paid much attention, and there are many methods proposed for the compensation. However, we should know the characteristics of the dispersion in order to get much higher efficient compensation algorithms. In this paper, we study the characteristics of the dispersion of optical fiber communication. According to the analysis to obtain the dispersion characteristics of optical fiber under different parameters. The main contribution of the paper is analysis of the characteristics of dispersion. And the remainder of the paper is shown as the following: The theoretical model is listed in section 2. Simulation and results are shown in section 3. And the conclusion is described in section 4 .

\section{The Theoretical Model}

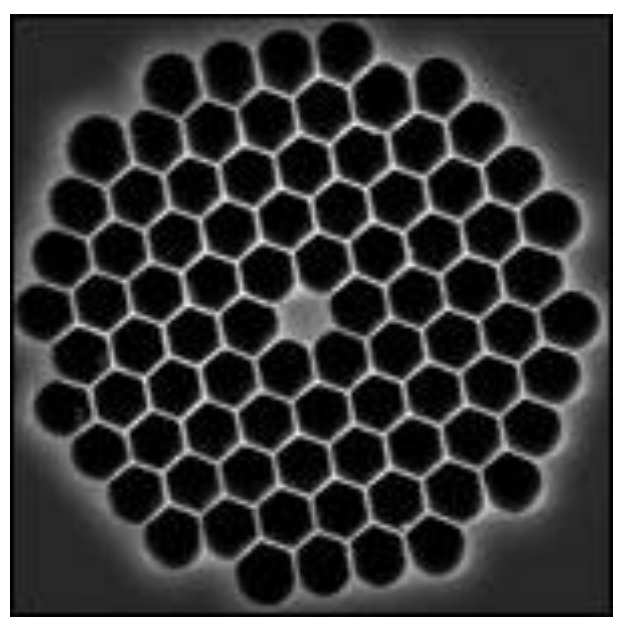

Figure 1. Structure of the Photonic Crystal Fiber 


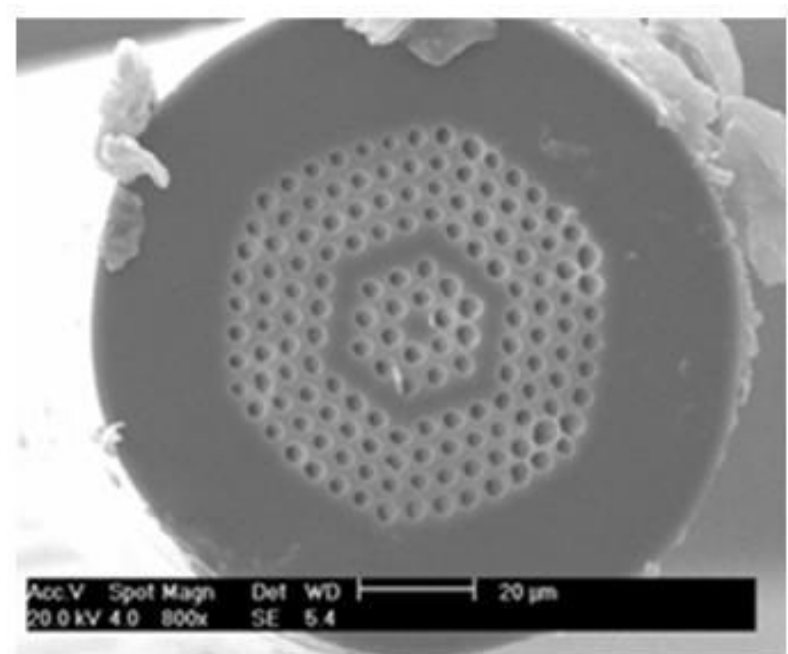

\section{Figure 2. Photonic Crystal Fiber under Scanning Electron Microscopy}

Figure 1 shows the structure of the photonic crystal fiber, and Figure 2 is a photograph of the photonic crystal fiber under scanning electron microscopy.

Fiber cross section is with infinite periodic hexagonal two-dimensional photonic crystal sequence and the center removing air holes to form the core. Characteristic equation of cladding space of photonic crystal fiber can be described as follows:

$$
\frac{I_{2}(w r)}{I_{1}(w r)}+\frac{1}{w r}+\frac{w r}{2}\left(1+\frac{n_{2}^{2}}{n_{1}^{2}}\right) g(u)+w r\left[\frac{1}{4}\left(1-\frac{n_{2}^{2}}{n_{1}^{2}}\right)^{2} g^{2}(u)+\frac{f(w, u)}{n_{1}^{2}}\right]^{\frac{1}{2}}=0
$$

Function

$$
\begin{gathered}
g(u)=\frac{1}{w r} \frac{J_{0}(u r) Y_{1}(u R)-Y_{0}(u r) J_{1}(u R)}{J_{1}(u r) Y_{1}(u R)-Y_{1}(u r) J_{1}(u R)}-\frac{1}{u^{2} r^{2}} \\
f(w, u)=\frac{1}{r^{4}}\left(\frac{1}{u^{2}}+\frac{1}{w^{2}}\right)\left(\frac{n_{2}^{2}}{u^{2}}+\frac{n_{1}^{2}}{w^{2}}\right) \\
m^{2}\left[\frac{1}{U^{2}}+\frac{1}{W^{2}}\right]\left[\frac{n_{1}^{2}}{U^{2}}+\frac{n_{2}^{2}}{W^{2}}\right]=\left[\frac{1}{U} \frac{J_{m}(U)}{J_{m}(U)}+\frac{1}{W} \frac{K_{m}(W)}{K_{m}(W)}\right] \times\left[\frac{n_{1}^{2} J_{m}(U)}{U \cdot J_{m}(U)}+\frac{n_{2}^{2}}{W} \frac{K_{m}(W)}{K_{m}(W)}\right]
\end{gathered}
$$

If we set $m=1$,

$$
\begin{gathered}
\frac{J_{0}(U)}{J_{0}(U)}=\frac{1}{U}+\frac{U}{2}\left(1+\frac{n_{2}^{2}}{n_{1}^{2}}\right)\left[\frac{1}{W^{2}}+\frac{K_{0}(W)}{W \cdot K_{1}(W)}\right]- \\
\frac{U}{2}\left(\sqrt{\left(1-\frac{n_{2}^{2}}{n_{1}^{2}}\right)^{2} \cdot\left[\frac{1}{W^{2}}+\frac{K_{0}(W)}{W \cdot K_{1}(W)}\right]^{2}+4 F(U, W)}\right) \\
F(U, W)=\left(\frac{1}{U^{2}}+\frac{1}{W^{2}}\right)\left(\frac{1}{U^{2}}+\frac{n_{2}^{2}}{n_{1}^{2}} \frac{1}{W^{2}}\right)
\end{gathered}
$$

Relation between $U, W$ and waveguide normalized frequency $V$ can be described as:

$$
U^{2}=\left(k^{2} n_{c}^{2} \cdot \beta_{c}^{2}\right) \cdot a_{e f f}^{2}
$$




$$
\begin{gathered}
W^{2}=\left(k^{2} n_{e f f}^{2} \cdot \beta_{c}^{2}\right) \cdot a_{e f f}^{2} \\
V^{2}=U^{2}+W^{2}=k^{2}\left(n_{c}^{2} \cdot n_{e f f}^{2}\right) \cdot a_{e f f}^{2}
\end{gathered}
$$

Where, $\beta_{c}$ is the propagation constant; $k$ is the optical wave vector; $n_{c}$ is the refractive index of the fiber core; $a_{e f f}$ is equivalent fiber core radius of photonic crystal fiber. If we calculate the numerical solution $U$ will be equal to $U(\omega)$. Then, we can get:

$$
\beta_{\mathrm{c}}(\omega)=\sqrt{k^{2} n_{c}^{2}(\omega)-U^{2}(\omega) / a_{\text {eff }}^{2}}
$$

Where, the effective fiber cladding index can be determined with the formula described before. Refraction index $n_{c}(\omega)$ of quartz material of the fiber core can be obtained by Sellmeier equation. Due to the characteristic function including Bessel function, it falls into the transcendental equations and numerical solution of $\beta_{c}$ with wavelength (or frequency) variation. In the paper, we will use Fourier series to fit the curve.

With the principle of waveguide, group velocity dispersion of the optical fiber $D$ can be described as:

$$
D=\frac{d \tau}{d \lambda}=-\frac{\omega^{2}}{2 \pi c} \frac{d^{2} \beta_{c}}{d \omega^{2}}
$$

In the formula described above, material dispersion, waveguide dispersion, and profile dispersion are all included, total dispersion type of single mode optical fiber with the incident light wavelength (or frequency) can be obtained according to the equation. Furthermore, dispersion slope $D$ of the optical fiber with wavelength can be obtained:

$$
D_{\text {slope }}=\frac{d D}{d \lambda}=-\frac{1}{\lambda^{2}} \frac{d D}{d \omega}
$$

Relationship between waveguide normalized frequency $V$ and wavelength can be described as:

$$
V=k \sqrt{\left(n_{\infty}^{2}-n_{e f f}^{2}\right)} a_{e f f}=\frac{2 \pi}{\lambda} \sqrt{\left(n_{\infty}^{2}-n_{\text {eff }}^{2}\right)} a_{\text {eff }}
$$

The value $V$ can be used to initially determine the single mode propagation range of optical fiber, and if $V \leq 21405$, it can be thought that just fundamental mode can be propagated in optical fiber.

For both scalar effective refractive index method and vector effective index method, a key parameter in the simulation of optical fiber dispersion is the equivalent core radius $a_{\text {eff }}$. There are several different methods for the determination of equivalent core radius. For example,

$$
a_{\text {eff }}=A
$$

Or

$$
a_{\text {eff }}=A-r
$$

Where, $\mathrm{A}$ is cladding air hole pitch, and $\mathrm{R}$ is the air hole radius. Determination methods as the following have been also adopted:

$$
a_{\text {eff }}=A / 2 \text {. }
$$

When the finite element method is applied, the effective core radius is about $0.64 A$. With different values of $d / A \leq 0.43$, the effective core radius is about $0.62 A \sim 0.63 A$. According to the analogy between photonic crystal fiber and step index fiber and considering the consistency of the two single mode propagation conditions, effective radius of core fiber should be $0.625 \mathrm{~A}$. Above all the situations and combined with different details, $a_{\text {eff }}$ is set with value of 0.64 . And $a_{\text {eff }}=0.64$ is the base in the paper.

Define nonlinear coefficient: 


$$
\gamma=\frac{n_{2} \omega_{0}}{\mathrm{cA}_{\mathrm{eff}}}=\frac{2 \pi}{\lambda} \frac{n_{2}}{A e_{f f}}
$$

Where, $n 2=2.76 \times 10^{-20} \mathrm{~m}^{2} / \mathrm{W}$, and it is the quartz nonlinear refractive index;

$A_{\text {eff }}$ Is the effective area of basic model of optical fiber.

$\omega_{0}$ Is the angular frequency

$c$ Is the light velocity in vacuum; $\lambda$ is the wavelength;

$A_{e f f}$ Can be used as an indicator of the nonlinear characteristics, and small $A_{\text {eff }}$ will enhance nonlinear effects.

$$
\mathrm{A}_{\mathrm{eff}}=\frac{\left(\iint_{s}\left|E_{t}\right|^{2} d x d y\right)^{2}}{\left(\iint_{s}\left|E_{t}\right|^{4} d x d y\right)}
$$

Where, $E_{t}$ is the transverse electric field vector, $\mathrm{S}$ is the cross section of the photonic crystal fiber.

$A_{\text {eff }}$ Depends on two factors, refraction difference between the core and cladding rate and the core size.

\section{Simulation and Results}

\subsection{Dispersion Characteristics}

Relationship between $A_{e f f}, \gamma, d$ and $\Lambda$ is shown in Figure.3 and Figure.4.

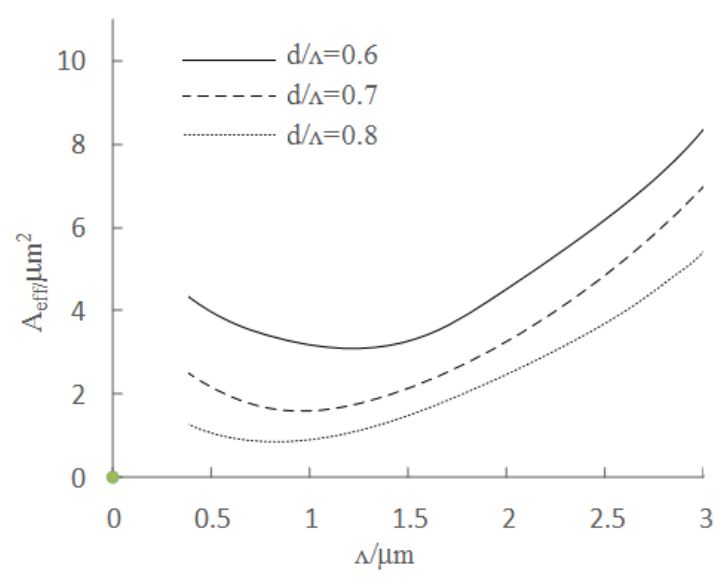

Figure 3. Relationship between ${ }^{A_{e f f}}, d$ and $\Lambda$ 


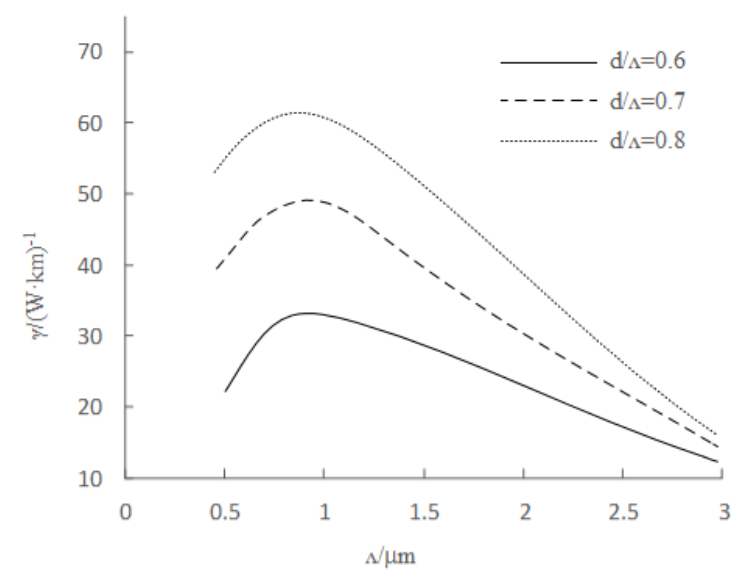

Figure 4. Relationship between $\gamma, d$ and $\Lambda$

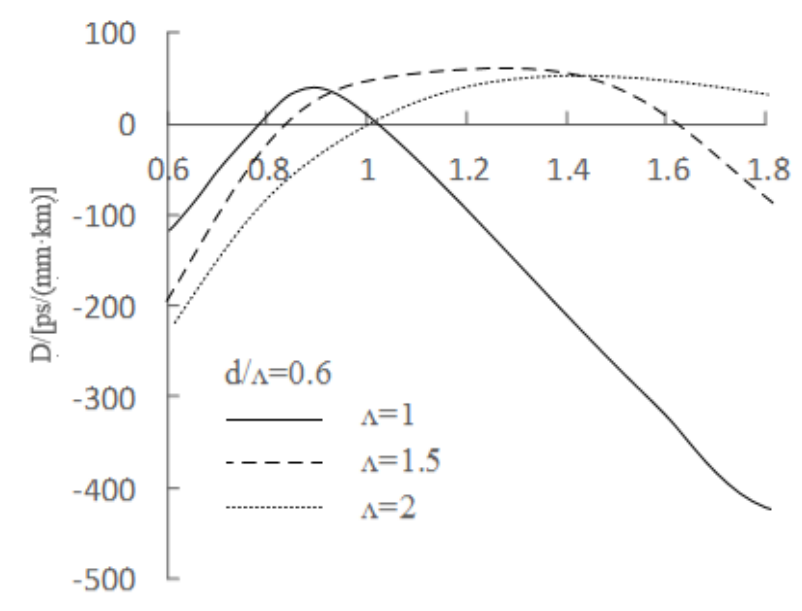

$\lambda \mu \mathrm{m}$

Figure 5. Relationship between $D$ and $\lambda$

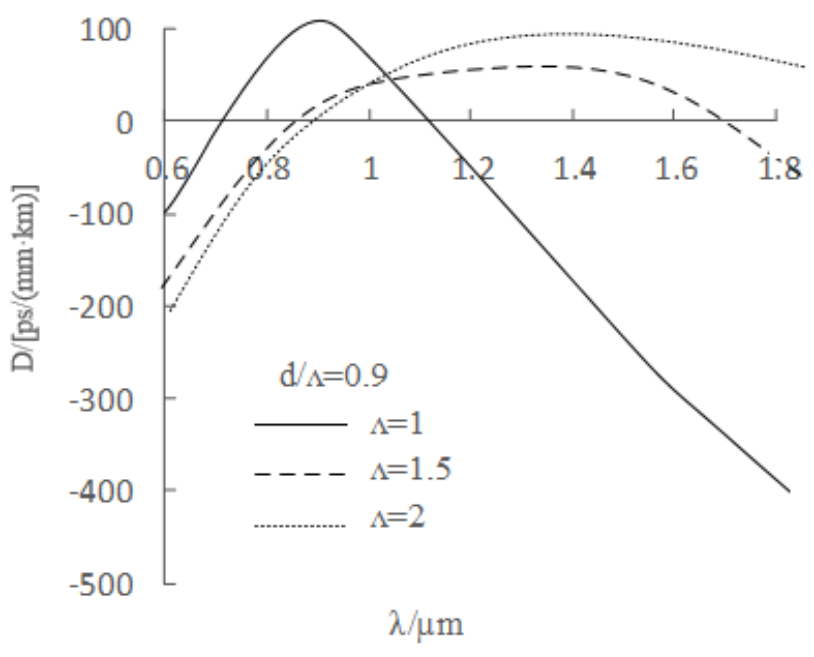

Figure 6. Relationship between $D$ and $\lambda$ 


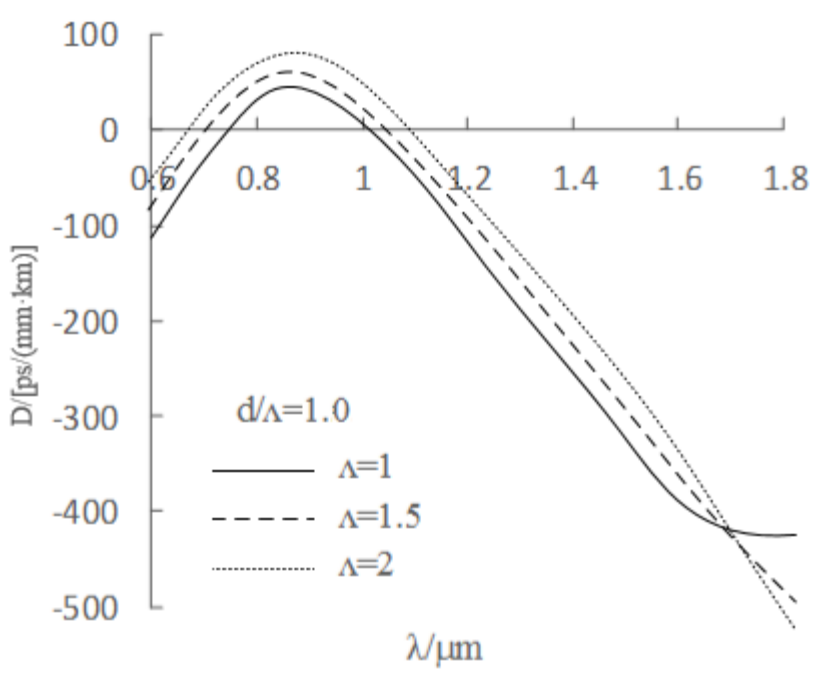

Figure 7. Relationship between $D$ and $\lambda$

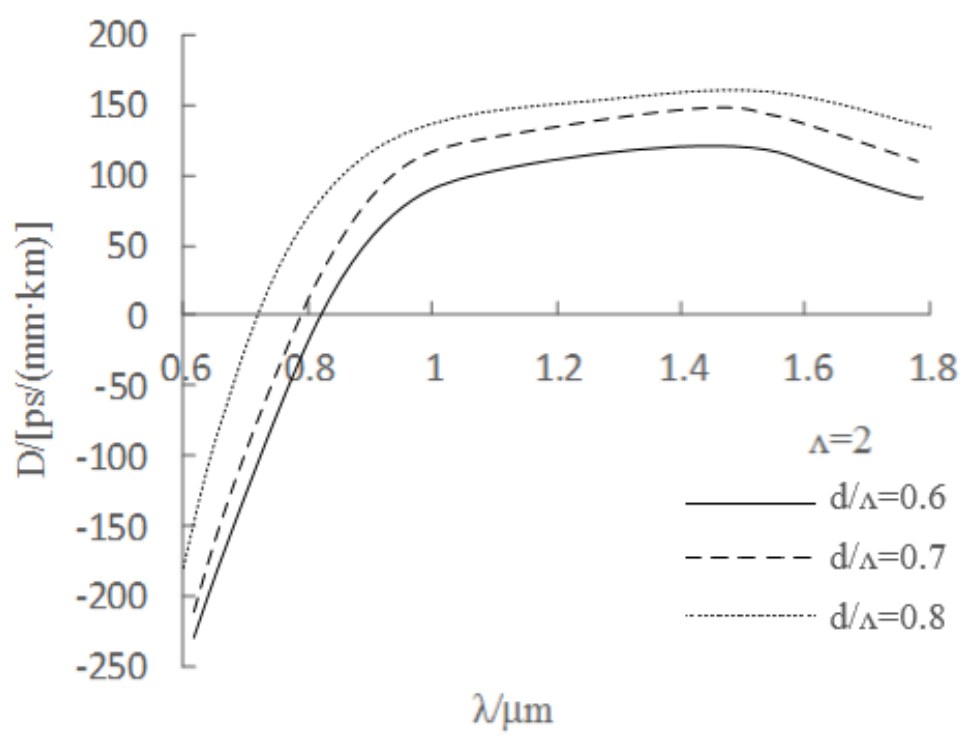

Figure 8. Relationship between $D$ and $\lambda$

From the figures we can see, each $d / \Lambda$ has a maximum value near $\lambda=1$. With the increasing of $d / \Lambda$, corresponding $\Lambda$ of $\gamma_{\max }$ will decrease. This shows that the mode limits ability of high nonlinear photonic crystal fiber is increased with the decrease of $\Lambda$. But when the value is reduced to about $1 \mu \mathrm{m}$, which is equivalent to the wavelength, mode field limit ability will decline and result in decreasing of nonlinear coefficient. This means that nonlinearity has a maximum value at about $1 \mu \mathrm{m}$ for the determined structure parameter. For different conditions, we can choose different structural parameters. And the nonlinear coefficient of ordinary single-mode fiber is $1 W^{-1} \cdot \mathrm{km}^{-1}$, which indicates that the high nonlinear photonic crystal fiber has strong nonlinear effects and it is the ideal nonlinear material.

When keep the $d / \Lambda$ as a constant and increasing the value of $\Lambda$, the first zero dispersion point shifts to long wavelength, and bending degree of dispersion curve decreases. This means the enhancement of the high nonlinear photonic crystal fiber mode field limiting ability, then dispersion will decrease greatly. This characteristic can be used in the dispersion compensation. When the $\Lambda$ reduces to below $1.5 \mu \mathrm{m}$, the second zero 
dispersion point will appear. With the decreasing of $\Lambda$ the second zero dispersion point will move forward to long wavelength. Then, if adjust the $\Lambda$, the second zero dispersion point will move to an adjacent area of wavelength with $1550 \mathrm{~nm}$, and it will satisfy the requirement of a continuous spectrum application.

When keep $\Lambda$ as the constant and increasing the value of $d / \Lambda$, the parameter $D$ will decrease and the first zero dispersion point will move the long wavelength. When the second zero point appears, second zero dispersion point will move the short wavelength. So we can design different structural parameters to modify the dispersion characteristics of highly nonlinear photonic crystal fiber to meet the demand of different applications.

\subsection{The Effective Refractive Index}

From figure.9, for a certain air hole spacing and aperture ratio of the photonic crystal fiber, the effective refractive index gradually decreases with the increase of wavelength. Under certain distance between the holes, the effective refractive ratio of the optical fiber decreases with the increasing of air hole diameter, which means the filling rate of air hole increases. This means that the mode field of light wave with longer wavelength in fiber is much closer to the air. It also shows photonic crystal fiber has low localization ability for the long wavelength light wave. This is due to the light field tends to spread in the medium in the short wavelength instead of air, and this will cause the decreasing of the effective refractive index with the decreasing of the wavelength, which is good for the transmission of the optical fiber with shorter wavelength.

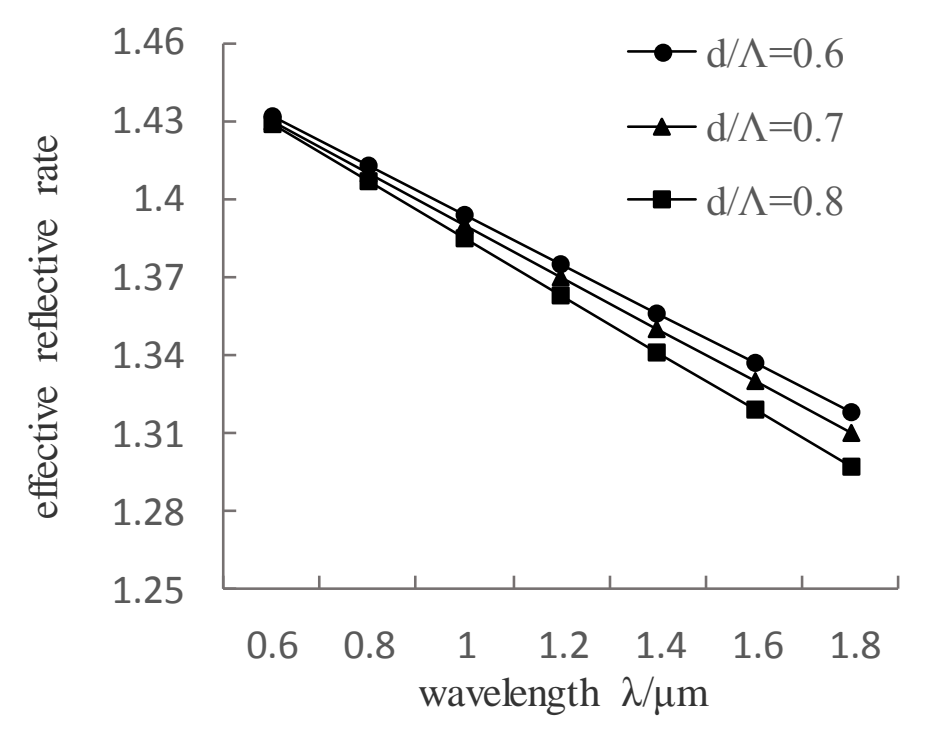

Figure 9. Effective Reflective Ratio when $\Lambda=1.0 \mu \mathrm{m}$ 


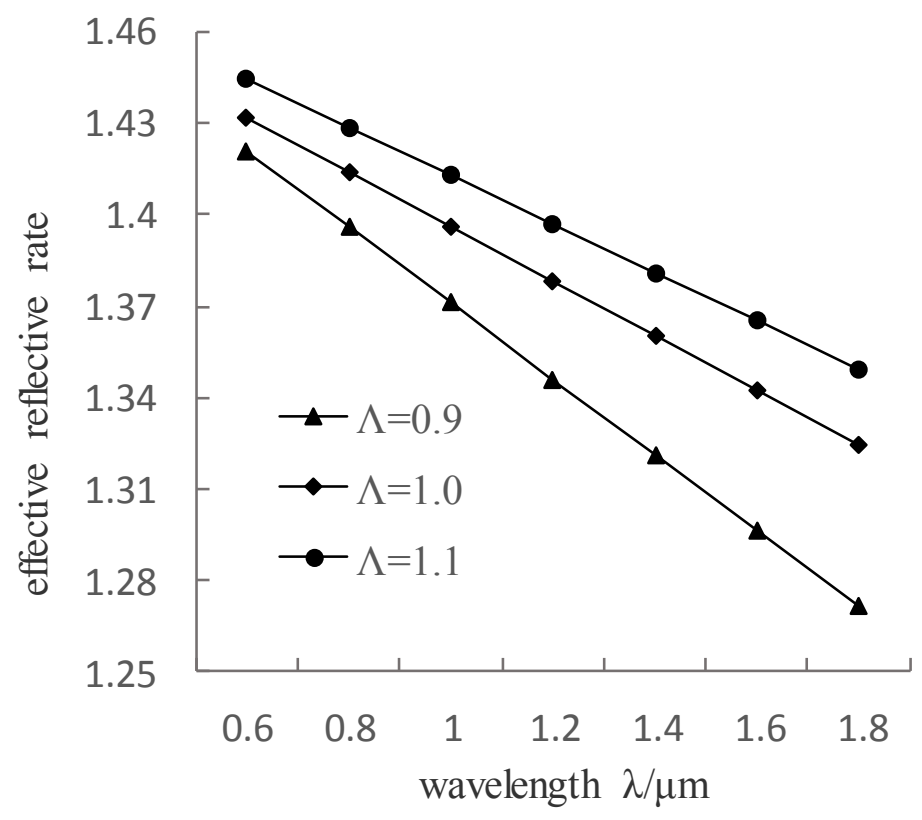

Figure 10. Effective Reflective Ratio when $\mathrm{d} / \Lambda=0.7 \mu \mathrm{m}$

From figure.10, with the increase of the distance between holes, the effective reflective index of the cladding of the optical fiber increases. With the decreasing of $\Lambda$, the effective reflective index of the cladding decreases, and mode field will be much closer to the air when the light wave is traveling in the optical fiber.

\subsection{The $D_{\text {slope }}$ characteristics}

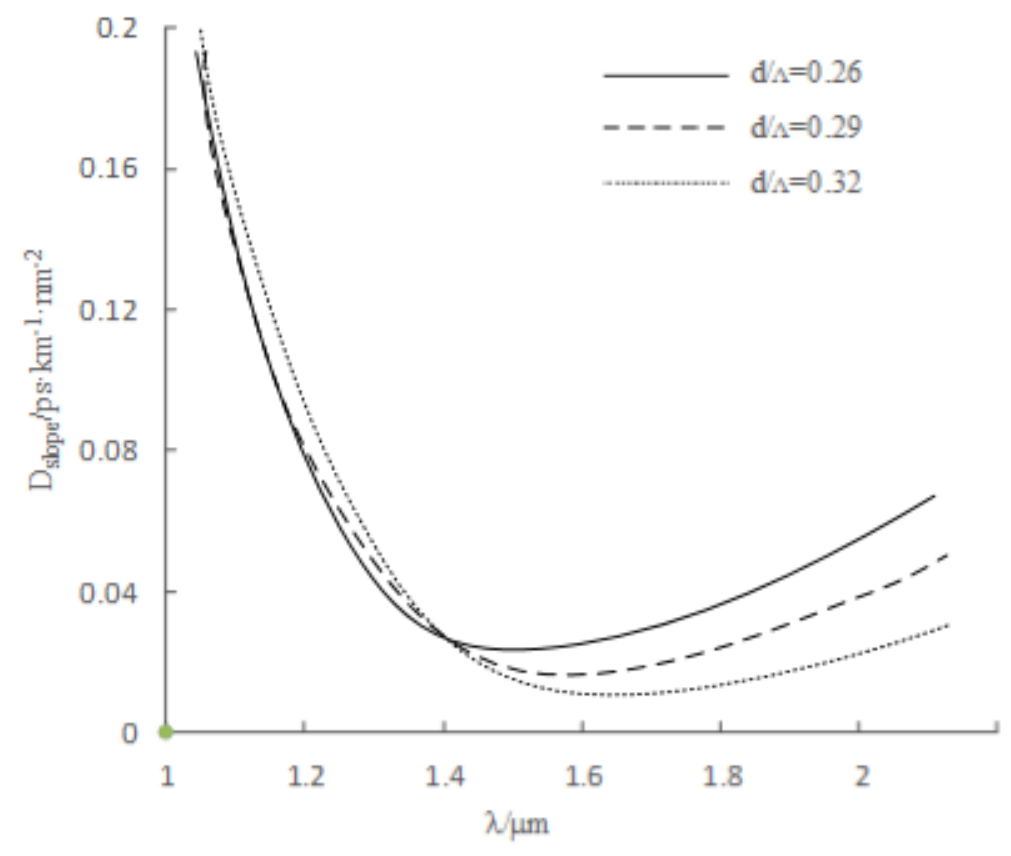

Figure 11. Dslope with $\Lambda=2.3 \mu \mathrm{m}$ 


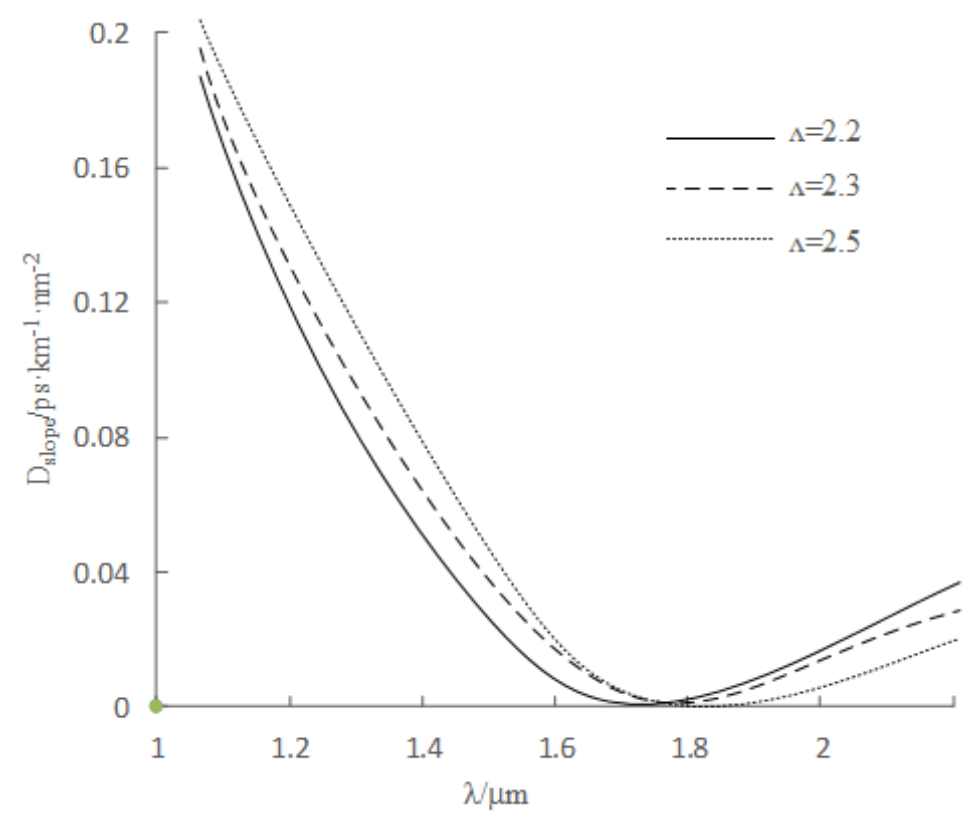

Figure 12. Dslope with $\mathrm{d} / \Lambda=0.6$

From figure.11 and figure.12, we can get some conclusions. Fiber of this series in the optical communication has a relatively small slope at WDM bands, which shows the characteristics of flattened dispersion.

When the fixed aperture ratio of and increasing distance between cladding air holes, the zero point of dispersion slope $D_{\text {slope }}$ will shift to longer wavelength. This is to say that fiber dispersion flattened area will move to the long wavelength. From corresponding wavelength of dispersion slope, we can get the results that the zero dispersion point moves to the long wavelength according to keeping the distance of $\Lambda$ as constant, increasing the diameter of air hole, maintaining the aperture ratio as constant, increasing the distance between air hole. That is to say that fiber dispersion flattened region moves to longer wavelength. So, we can flexibly change the optical fiber dispersion flatted region according to verify the structure.

\section{Conclusions}

Dispersion is widely existed in the optical fiber and the reason is also various, such as material dispersion, waveguide dispersion, mode dispersion, and so on. It will lead to the signal waveform distortion and pulse broadening and then limit the transmission capacity and transmission bandwidth in the optical fiber. With the rapid development, transmission rate can reach dozens or even hundreds of $\mathrm{Gb} / \mathrm{s}$, and it may lead to many problems.

In order to solve the problem and improve the data transmission rate, we should first know the characteristics of the dispersion in the optical fiber. Optical fiber systems with better performance will be designed and produced.

In the paper, appropriate methods are adopted to analyze the nonlinear photonic crystal fiber. With the adjusting of the structure parameters, we can design a highly nonlinear photonic crystal fiber with different dispersion characteristics. The simulation and analysis results can provide the theoretical basis for material selection and application for optical fiber device. 


\section{References}

[1] K. Ivan, T. Li, A. E. Willner eds., "Optical Fiber Telecommunications Volume VIA: Components and Subsystems", Academic press, (2013).

[2] R. Essiambre, G. Kramer, P. J. Winzer, G. J. Foschini, B. Goebel, "Capacity limits of optical fiber networks. Lightwave Technology", vol. 28, no. 4, (2010), pp. 662-701.

[3] K. Predehl, G. Grosche, S. M. F. Raupach, S. Droste, O. Terra, J. Alnis, H. Schnatz, "A 920-kilometer optical fiber link for frequency metrology at the 19th decimal place”, Science, vol. 336, no. 6080, (2012), pp. 441-444.

[4] T. J. Kippenberg, R. Holzwarth, \& S. A. Diddams, "Micro resonator-based optical frequency combs", Science, vol. 332, no. 6029, (2011), pp. 555-559.

[5] A. E. N. A. Mohamed, M. M. El-Halawany, Z. Rashed, A. Nabih, M. S. Tabbour, "High Transmission Performance of Radio Over Fiber Systems over Traditional Optical Fiber Communication Systems Using Different Coding Formats for Long Haul Applications", Nonlinear Optics, Quantum Optics: Concepts in Modern Optics, vol. 44, no. 1, (2012).

[6] F. Poletti, X. Feng, G. M. Ponzo, M. N. Petrovich, W. H. Loh, D. J. Richardson, "All-solid highly nonlinear singlemode fibers with a tailored dispersion profile", Optics express, vol. 19, no. 1, (2011), pp. 66-80.

[7] H. L. Rogers, C. Holmes, J. C. Gates, P. G. R. Smith, "Analysis of dispersion characteristics of planar waveguides via multi-order interrogation of integrated Bragg gratings", Photonics Journal, IEEE, vol. 4, no. 2, (2012), pp. 310-316.

[8] H. L. Rogers, C. Holmes, J. C. Gates, P. G. Smith, "Direct refractive index measurement technique to observe waveguide dispersion characteristics of short waveguides utilizing the higher order modes of integrated Bragg grating structures", In The European Conference on Lasers and Electro-Optics (p. CE1_1). Optical Society of America, (2011).

[9] T. Godin, Y. Combes, R. Ahmad, M. Rochette, T. Sylvestre, J. M. Dudley, "Normal dispersion modulation instability in an As2Se3 chalcogenide hybrid microwire", SPIE Photonics Europe, International Society for Optics and Photonics, (2014), pp. 91360P-91360P.

[10] Marra G., R. Slavík, H. S. Margolis, S. N. Lea, P. Petropoulos, D. J. Richardson, P. Gill, "Highresolution microwave frequency transfer over an 86-km-long optical fiber network using a mode-locked laser", Optics letters, vol. 36, no. 4, (2011), pp. 511-513.

[11] M. Brodsky, E. C. George, C. Antonelli, M. Shtaif, "Loss of polarization entanglement in a fiber-optic system with polarization mode dispersion in one optical path", Optics letters, vol. 36, no. 1, vol. 43-45, (2011)

[12] R. Ryf, S. Randel, A. H. Gnauck, C Bolle, A. Sierra, S. Mumtaz, R. Lingle, "Mode-division multiplexing over $96 \mathrm{~km}$ of few-mode fiber using coherent 66 MIMO processing", Lightwave Technology, Journal of, vol. 30, no. 4, (2012), pp. 521-531.

[13] Y. Choi, C. Yoon, M. Kim, T. D. Yang, C. Fang-Yen, R. R. Dasari, W. Choi, "Scanner-free and widefield endoscopic imaging by using a single multimode optical fiber", Physical review letters, vol. 109, no. 20, (2012), pp. 203901.

[14] M. M. Haque, M. S. Rahman, M. S. Habib, S. M. A. Razzak, "Design and characterization of single mode circular photonic crystal fiber for broadband dispersion compensation", Optik-International Journal for Light and Electron Optics, vol. 125, no. 11, (2014), pp. 2608-2611.

[15] J. Sakaguchi, Y. Awaji, N. Wada, A. Kanno, T. Kawanishi, T. Hayashi, M. Watanabe, "109-Tb/s (7x97x172-Gb/s SDM/WDM/PDM) QPSK transmission through 16.8-km homogeneous multi-core fiber", Optical Fiber Communication Conference Optical Society of America, (2011), p. PDPB6.

[16] S. Spolitis, G. Ivanovs, "Extending the reach of DWDM-PON access network using chromatic dispersion compensation", Communication Technologies Workshop (Swe-CTW), 2011 IEEE Swedish, (2011) pp. 29-33.

[17] L. B. Du, A. J. Lowery, "Improved single channel back propagation for intra-channel fiber nonlinearity compensation in long-haul optical communication systems", Optics Express, vol. 18, no. 16, (2010), pp. 17075-17088.

[18] P. Li, J. Shuisheng, X. Zenghua, N. Tigang, L. Tangjun, "Dispersion compensation optical fiber grating with low ripple coefficient”, Acta Optica Sinica, vol. 22, no. 3, (2002), pp. 336-339.

[19] M. Brodsky, E. C. George, C. Antonelli, M. Shtaif, "Loss of polarization entanglement in a fiber-optic system with polarization mode dispersion in one optical path", Optics letters, vol. 36, no. 1, 43-45, (2011)

[20] B. N. Hu, W. Jing, W. Wei, R. M. Zhao, "Analysis on Dispersion Compensation with DCF based on Optisystem", Industrial and Information Systems (IIS), 2010 2nd International Conference, vol. 2, (2010), pp. 40-43.

[21] X. Zhou, E. F. Mateo, G. Li, "Fiber nonlinearity management-from carrier perspective", National Fiber Optic Engineers Conference, Optical Society of America, (2011), p. NThB4. 
[22] V. Veljanovski, V. Sleiffer, D. Borne, J. Capasso, H. Kuluslu, J. Seixas, H. Waardt, "125-Gb/s CPQPSK field trial over $4108 \mathrm{~km}$ of installed submarine cable", Optical Fiber Communication Conference, OSA Technical Digest (CD)(Optical Society of America, 2011), paper PDPD3, (2011)

[23] A. Eghbali, H. Johansson, O. Gustafsson, S. J. Savory, "Optimal Least-Squares FIR Digital Filters for Compensation of Chromatic Dispersion in Digital Coherent Optical Receivers", Journal of Lightwave Technology, vol. 32, no. 8, (2014), pp. 1449-1456.

[24] P. J. Winzer, "High-spectral-efficiency optical modulation formats", Lightwave Technology, Journal of, vol. 30, no. 24, (2012), pp. 3824-3835.

[25] Z. Jia, J. Yu, H. C. Chien, Z. Dong, D. D. Huo, "Field transmission of $100 \mathrm{G}$ and beyond: multiple baud rates and mixed line rates using Nyquist-WDM technology", Lightwave Technology, Journal of, vol. 30, no. 24, (2012), pp. 3793-3804.

\section{Authors}

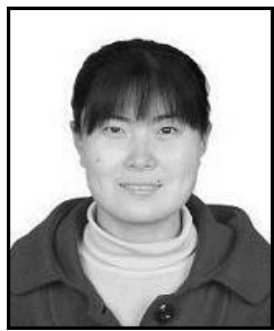

Ni Yan Rong, 1981.7, Xinxiang, Henan, P.R. China; She is a lecturer in college of engineering science, Henan Mechanical and Electrical Engineering College, Henan, China. Her scientific interests are wire and cable manufacturing technology.

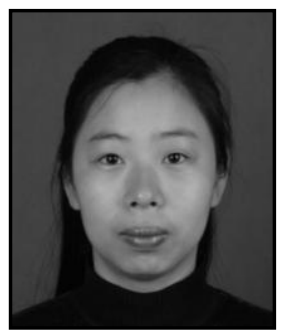

Min Ru,1979.12, Xinxiang, Henan, P.R. China; She is a lecturer in college of engineering science, Henan Mechanical and Electrical Engineering College, Henan, China. Her scientific interests are Optical fiber communication technology. 